\title{
Phase-Averaged Method Applied to Periodic Flow Between Shrouded Co-Rotating Disks
}

\author{
Shen-Chun Wu \\ National Defense University, Tauyuan, Taiwan, Republic of China \\ Yau-Ming Chen \\ Department of Mechanical Engineering, National Taiwan University, Taipei, Taiwan, \\ Republic of China
}

This study investigates the coherent flow fields between co-rotating disks in a cylindrical enclosure. By using two laser velocimeters and a phase-averaged technique, the vortical structures of the flow could be reconstructed and their dynamic behavior was observed. The experimental results reveal clearly that the flow field between the disks is composed of three distinct regions: an inner region near the hub, an outer region, and a shroud boundary-layer region. The outer region is distinguished by the presence of large vortical structures. The number of the vortical structures corresponds to the normalized frequency of the flow.

Keywords Phase average method; Co-rotating disks; Laser doppler velocimetry

The fluid motion in the space between a pair of co-rotating disks is of considerable importance to hard disk drives, which are extensively used as data storage devices in computers. This is because the large-scale oscillating flow structure induced by rotation of disks and vortexes shedding from obstructions contribute to alignment inaccuracies and, hence, to the degraded performance of the read-write magnetic heads that are supported at submicron distance from the rotating disk surfaces.

Lennemann (1974) visualized the flow in an axisymmetric cylindrical shroud by using aluminum powder as the tracer. Without an obstruction, Lennemann observed a laminar solid body rotation core region with an unsteady polygon lobedshaped boundary. The core was reported to rotate at $80 \%$ of

Received 15 January 2001; accepted 15 May 2001

Address correspondence to Professor Yau-Ming Chen, Department of Mechanical Engineering, National Taiwan University, Taipei, Taiwan 106, Republic of China. E-mail: ymchen@ccms.ntu.edu.tw the disk rotation rate and highly turbulent flow was observed nearby the shroud. Using a similar water flow apparatus and visualization technique, Kaneko, Oguchi, and Hoshiya (1977) observed a laminar core that extended from the hub out to the middle radii of the disks. An outer turbulent region surrounded the bumpy laminar core. Abrahamson et al. (1988, 1989, 1991) employed bromothymol blue dye and a hydrogen bubble technique for flow visualization and reported the existence of a center core region in solid body rotation and the presence of circumferentially periodic flow structures with a strong component of axial vorticity near the shroud, processing at roughly $75 \%$ of the disk rotation rate.

Schuler et al. (1990) also employed a LDV to measure the mean and rms of the circumferential velocity component between the central pair of four co-rotating disk flow. As being normalized with the local disk surface velocity, the mean and rms velocity measured by Tzeng, Munce, and Crawforth (1991) at $R e=7.9 \times 10^{4}$ show fairly good agreement with the data measured by Schuler et al. at $R e=8.9 \times 10^{4}$. The time sequence measured by Schuler et al. displays distinct sinusoidallike oscillations. By using a copper-vapor laser for illuminating aerosol flow between co-rotating disks and a high resolution CCD camera, Tzeng, Munce, and Crawforth (1991) were able to report on the number of sides of the polygonal interface at high disk speeds, and confirmed the agreement of the inscribing radius of the polygonal structure, evaluated by flow visualization.

Tzeng and Humphrey (1991) used a laser-Doppler velocimetry to measure the mean and rms of the circumferential velocity components between co-rotating disks in an axisymmetric enclosure. Their normalized radial profiles of the circumferential mean velocity, showed two flow regions separated by a dimensionless radius $r^{*}=0.55$. These consist of an inner region $\left(r^{*}<0.55\right)$ where the fluid velocity is within $10 \%$ of the disk velocity and an outer region $\left(r^{*}>0.55\right)$ where the normalized 
velocity decreased linearly to $\cong 63 \%$ of the disk velocity at the disk edge $\left(r^{*}=1\right)$.

By illuminating the micro-sized mineral oil droplets with a laser sheet, Humphrey and Gor (1993) visualized the crossstream flow in an experimental apparatus similar to that used by Schuler et al. (1990). Humphrey, Schuler, and Weber (1995) numerically investigated the unsteady streamlined motion of a constant property fluid in the unobstructed space between disks co-rotating in a fixed cylindrical enclosure using a time-explicit algorithm.

Although efforts of the experimental measurements have been made in the above-mentioned studies, the unsteady aspects of the oscillatory flow structure induced by shrouded co- rotating disks are still not well informed. Previous studies focused on the flow visualization methods. Quantitative measurement data are therefore still lacking. In this regard, a measuring technique employing two Laser Doppler Velocimeters (LDV) together with phase-averaged method was developed to accomplish time-resolved measurements of periodic flow structures induced by shrouded corotating disks in the present study.

\section{EXPERIMENTAL APPARATUS AND TECHNIQUES}

\section{Apparatus and LDV Instrumentation}

The experimental test section and its associated coordinate system are schematically shown in Figure 1. Two rigid disks of

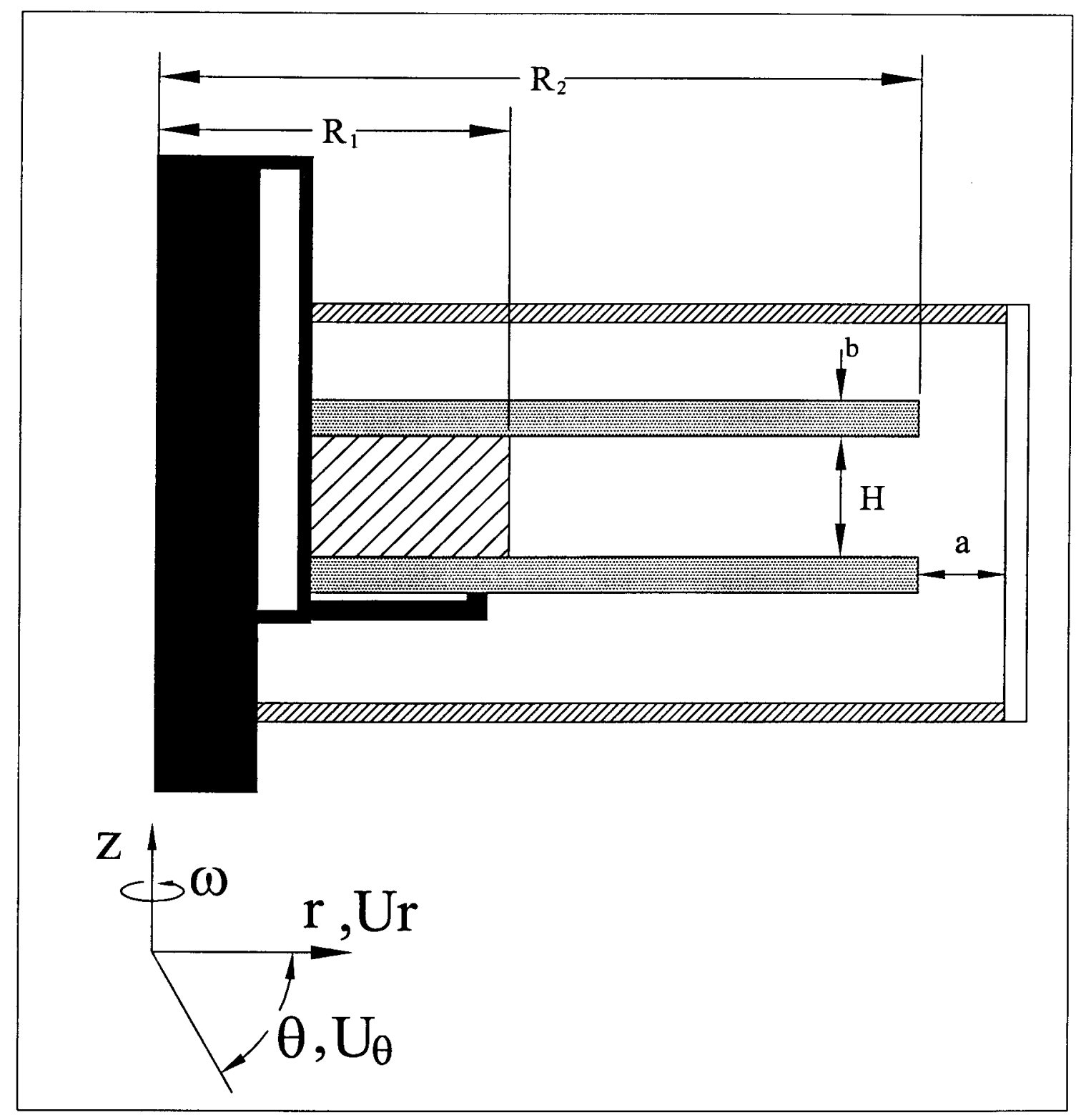

FIGURE 1

Test section and coordinate system. 
outer radius $R_{2}=1875 \mathrm{~mm}$ were concentrically mounted on a hub. The disks were both made of transparent Plexiglas and of thickness $b=10 \mathrm{~mm}$. A spacer ring of height $H=23.2 \mathrm{~mm}$ and radius $R_{1}=59.4 \mathrm{~mm}$ was used to separate the disks in the spindle axis direction ( $z$ direction). The test section shroud was made of transparent Plexiglas ${ }^{\circledR}$ of rectangular parallelepiped outer shape and was machined to achieve a cylindrically axisymmetric inner wall with radius $R_{2}+a=198.5 \mathrm{~mm}$. The base and the enclosure top cover of the test section were also made of Plexiglas ${ }^{\circledR}$ to allow the passage of laser light. Pure water was used as the working fluid in the experiment. The $\mathrm{MgO}$ particles with a diameter of about $1.2 \mu \mathrm{m}$ were used as seeding material for the LDV measurements.

The principles and practice of laser-Doppler anemometry have been introduced elsewhere, like Durst, Melling, and
Whitelaw (1981). Only the LDV system with direct relevance to this study was mentioned here. Two laser velocimeters, LDV and PDPA, were used in the present work in order to carry out the measurements with phase-averaged technique. The optics and electronic setup were schematically shown in Figure 2. The TSI Model 9201 Colorburst Multicolor Beam Separator converts an argon-ion laser (Coherent Innova 90, 2W) beam into four laser beams to perform laser Doppler velocimetry. The conversion produces two pairs of shifted and unshifted output beams: two green beams at $514.5 \mathrm{~nm}$, and two blue beams at $488.0 \mathrm{~nm}$. A Bragg cell was used to shift the frequency of each color as $40 \mathrm{MHz}$ to eliminate directional ambiguity. The Model 9271 Coupler launches light into a single-mode polarizationpreserving fiber. The resulting two pairs of beams were then passed through a $350 \mathrm{~mm}$ focal length lens. The focused beams

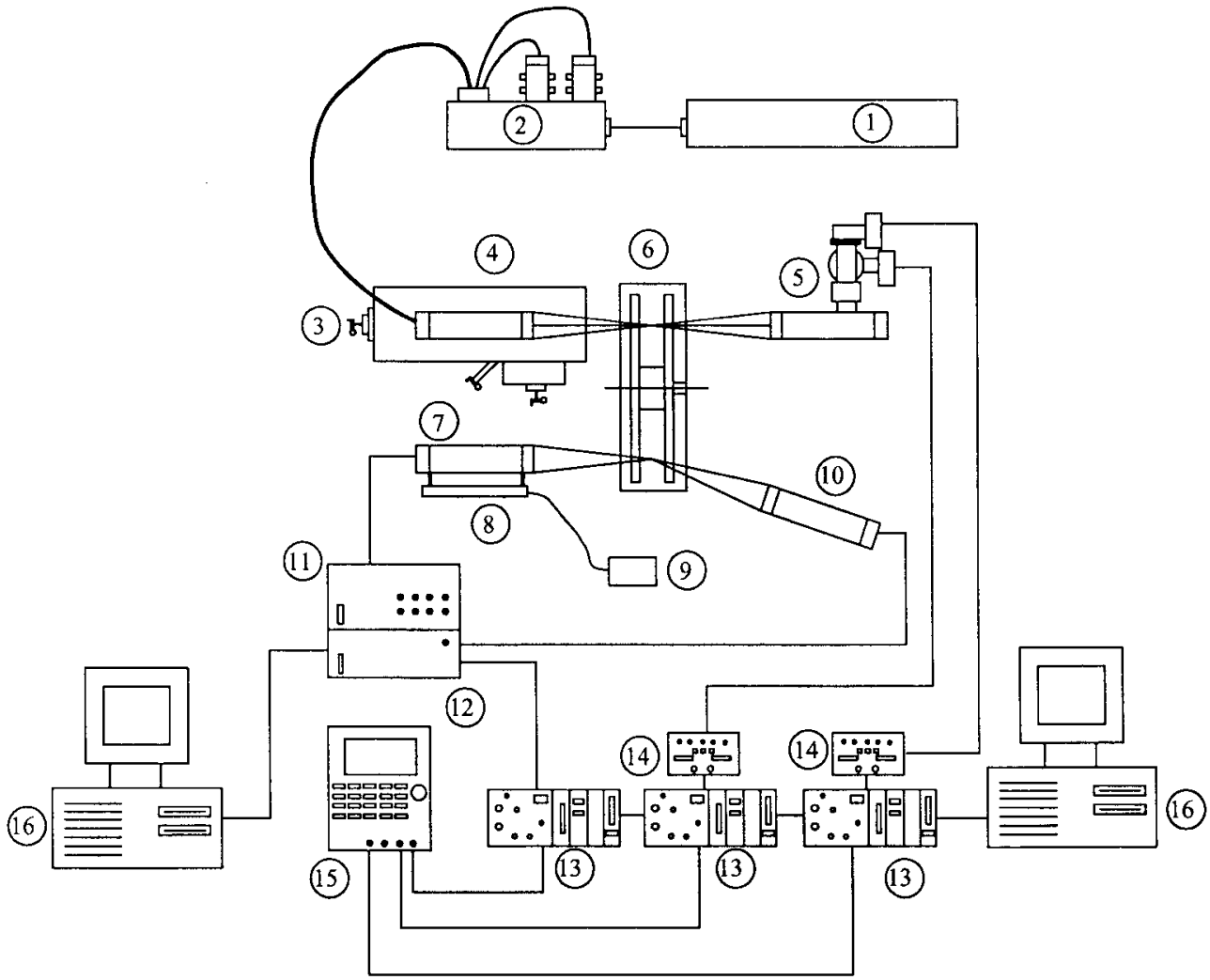
(1) Ar Ion Laser
(5) LDV Receiver
(9) Laser Power Supply
(13) LDV Counter
(2) Colorburst
(6) Test Section
(10) PDPA Receiver
(14) Downmixer
(3) Milling Machine
(7)
PDPA Transmitt
(11)
Motor Controller
(15) Oscilloscope
(4) LDV Probe
(8) $\mathrm{He}-\mathrm{Ne}$ Laser
(12) PDPA Counter
(16) Computer

FIGURE 2

2 LDV systems. 
intersected between the pair of the disks, giving a probe volume with dimensions of $0.52 \mathrm{~mm} \times 0.10 \mathrm{~mm}$ at the $e^{-2}$ extent of light intensity. The light scattered from seeding particles was collected by a receiving optical package, which reflects the collected light into a photomultiplier. The detected signal was electrically downmixed to the approximate frequency shift ( $0.1 \mathrm{Mhz} \sim 10 \mathrm{Mhz}$ in this experiment). TSI counter processors with $1 \mathrm{~ns}$ resolution were then used to process the Doppler signals. The Doppler signals were monitored on an oscilloscope, and the digital output of the counter processors was fed directly to a personal computer for storage and further analysis.

The PDPA system consists of five major components consisting of transmitter, receiver, signal processor, motor controller box, and computer. The Aerometrics model XMT-1100-4S transmitter was used to generate two equal intensity laser beams and focus them to an intersection point, which forms the measurement region. The transmitter contains a Spectra-Physics model SP-106, $10 \mathrm{~mW}$, polarized helium-neon laser. Optical components within the transmitter serve to focus, partition, and collimate the laser beam. A radial diffraction grating beam splitter is mounted on to the shaft of a high-speed DC motor. Rotation of the grating produces a frequency differential between the diffracted beams, which is used to produce a frequency shift on measured particle signals. The receiver model RCA-2100 collected the light scattered from seed particles. The receiver was then connected to the PDP-3100 signal processor with $2 \mu \mathrm{s}$ resolution to process the Doppler signals. The analog output of PDP-3100 signal processor was transmitted to the TSI counter processor as input to offer the phase reference.

\section{Measurement Technique and Data Reduction}

The rotational speed of disks was fixed at $266 \mathrm{rpm}$ in this study. By using the radius of the disk as the characteristic length and the disk tip speed as the reference velocity, the Reynolds number was calculated to be about $R e=1.06 \times 10^{6}$. The dimensionless axial distance of disks was fixed at $S=0.12$. Experiments were also carried out at $S=0.05$ for comparison. As shown in Figure 2, the PDPA probe was fixed at a specific position $\left(r^{*}=0.72, z / H=0.5\right)$. To investigate the vortical structures, all the measurements were made in a quarter of the center plane between the two co-rotating disks. The grids in the measuring domain were totally 4,096 points. As the measurements ran, the PDPA probe was kept at the specific position as a phase reference point, while the probe of LDV was translated in the measurement domain by the transverser. Doppler burst signals detected from PDPA and LDV were transferred to the LDV counters, which worked as coincident mode under the control of TSI MI-990 interface module. A coincident time window length $(0.5 \mathrm{~ms})$ was set in the MI-990 interface module to test the synchronization of burst signals detected by PDPA and LDV. The burst signals were processed to compute the Doppler shift frequency, as the time interval of signals detected by PDPA and LDV was lower than the coincident time window length. Data of Doppler shift frequency was then transferred to a personal computer via a DMA interface card. The velocity values were further calculated and then the time sequence was stored. Coincident data rate was kept at about $2000 \mathrm{~Hz}$ as the measurements were made, which were high enough to resolve the periodic motion of vortical structure with $9.9 \mathrm{~Hz}$ of frequency in the present study. During each measurement, more than 20,000 samples of time sequence of reference point (measured by PDPA) and measuring points were collected. All of the time histograms of velocity, whether circumferential or radial component, were measured with respect to the same phase reference point. The phase-averaged method was shown schematically in Figure 3. A cycle was built in two adjacent peak values of phase reference signal, and then, was divided into ten phases of the same time interval. The time series measured by LDV corresponding to each phase was sampled and averaged, respectively.

In order to investigate periodic flow fields, a decomposition of velocity proposed by Hussain and Reynolds (1970) and Reynolds and Hussain (1972) was used in analyzing the organized wave in turbulent shear flow. The oscillatory flow of co-rotating disks can be taken as combination of global mean components $\bar{u}$, a periodic mean $\tilde{u}$, and a random component $u^{\prime}$. By definition, the instantaneous velocity is the sum

$$
u=\bar{u}+\tilde{u}+u^{\prime}
$$

According to an analysis by Reynolds and Hussain (1972), the momentum equation that taking average at constant phase has

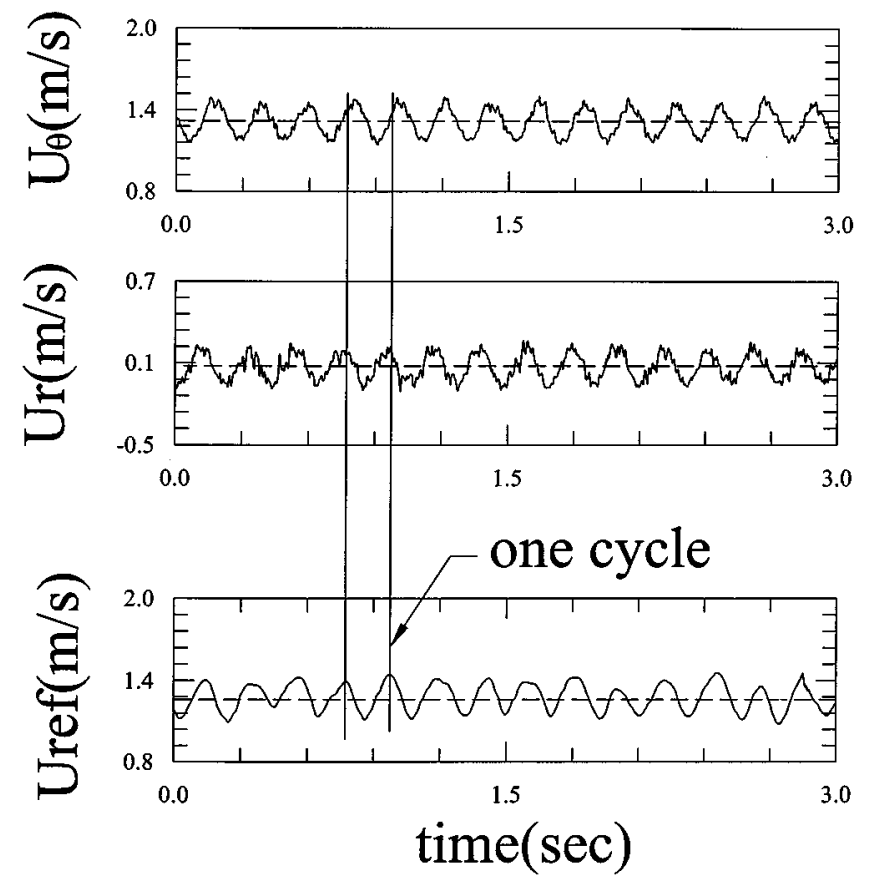

FIGURE 3

Typical time records of the circumferential and radial velocity at $r^{*}=0.395$. 
the form,

$$
\rho \frac{\partial\left\langle u_{i}\right\rangle}{\partial t}+\rho\left\langle u_{j}\right\rangle \frac{\partial\left\langle u_{i}\right\rangle}{\partial x_{j}}=-\frac{\partial\langle p\rangle}{\partial x_{i}}+\mu \frac{\partial^{2}\left\langle u_{i}\right\rangle}{\partial x_{k}^{2}}-\rho \frac{\partial\left(u_{i}^{\prime} u_{j}^{\prime}\right)}{\partial x_{j}}
$$

The momentum equation for the global mean flow has the form,

$$
\rho \bar{u}_{j} \frac{\partial \bar{u}_{i}}{\partial x_{j}}=-\frac{\partial p}{\partial x_{i}}+\mu \frac{\partial^{2} \bar{u}_{i}}{\partial x_{k}^{2}}-\rho \frac{\partial\left(\overline{\left\langle u_{i}^{\prime} u_{j}^{\prime}\right\rangle}+\overline{\tilde{u}_{i} \tilde{u}_{j}}\right)}{\partial x_{j}}
$$

Due to space limitation, only the results of global mean components are discussed in the following.

Bias of calculating global mean velocity was corrected by the method provided by McLaughlin and Tiederman (1973). Calibration was carried out in the test section with an oscilloscope and the maximum velocity bias was estimated to be less than $2 \%$. The statistical uncertainty for phase-average velocity was estimated to be within $4.5 \%$. Typical value was about $1.2 \%$.

\section{RESULTS AND DISCUSSION}

\section{Mean Velocity Profiles}

Figure 4 shows the normalized mean circumferential velocity component measured as a function of radial position along the symmetric plane midway between the two disks $(z / H=0.5)$. The mean values have been normalized by the local disk speed. A horizontal dashed line was plotted to represent the local disk speed $U / r \omega=1$. The dashed line manifests the solid body rotation. It is found in the figure that the velocity of fluid begins to deviate from solid body rotation at the position of about $r^{*}=0.35$ in the present study. Included for comparison are also the results measured by Abrahamson, Eaton, and Koga (1989) $\left(R e=4.5 \times 10^{5}\right)$ and Tzeng, Munce, and Crawforth (1991) $\left(R e=7.9 \times 10^{4}\right)$. The circumferential velocity begins to deviate from solid body rotation nearly at the same dimensionless radial position of about 0.45 in the results measured by Abrahamson, Eaton, and Koga (1989) and Tzeng, Munce, and Crawforth (1991). The reason that causes the difference is the larger axial distance of disks in the present work. The dimensionless axial distance by Abrahamson, Eaton, and Koga (1989) and Tzeng, Munce, and Crawforth (1991) was fixed at $S=0.05$ while the value adopted in the present study was $S=0.12$. The axial distance of disks by Abrahamson, Eaton, and Koga (1989) and Tzeng, Munce, and Crawforth (1991) were so small as to prevent the inflow with momentum deficit from penetrating larger distance inside. For comparison, the experiments were also performed at $S=0.05$. As can be seen, good agreement can be found between the present results and that of Abrahamson, Eaton, and Koga (1989) and Tzeng, Munce, and Crawforth (1991).

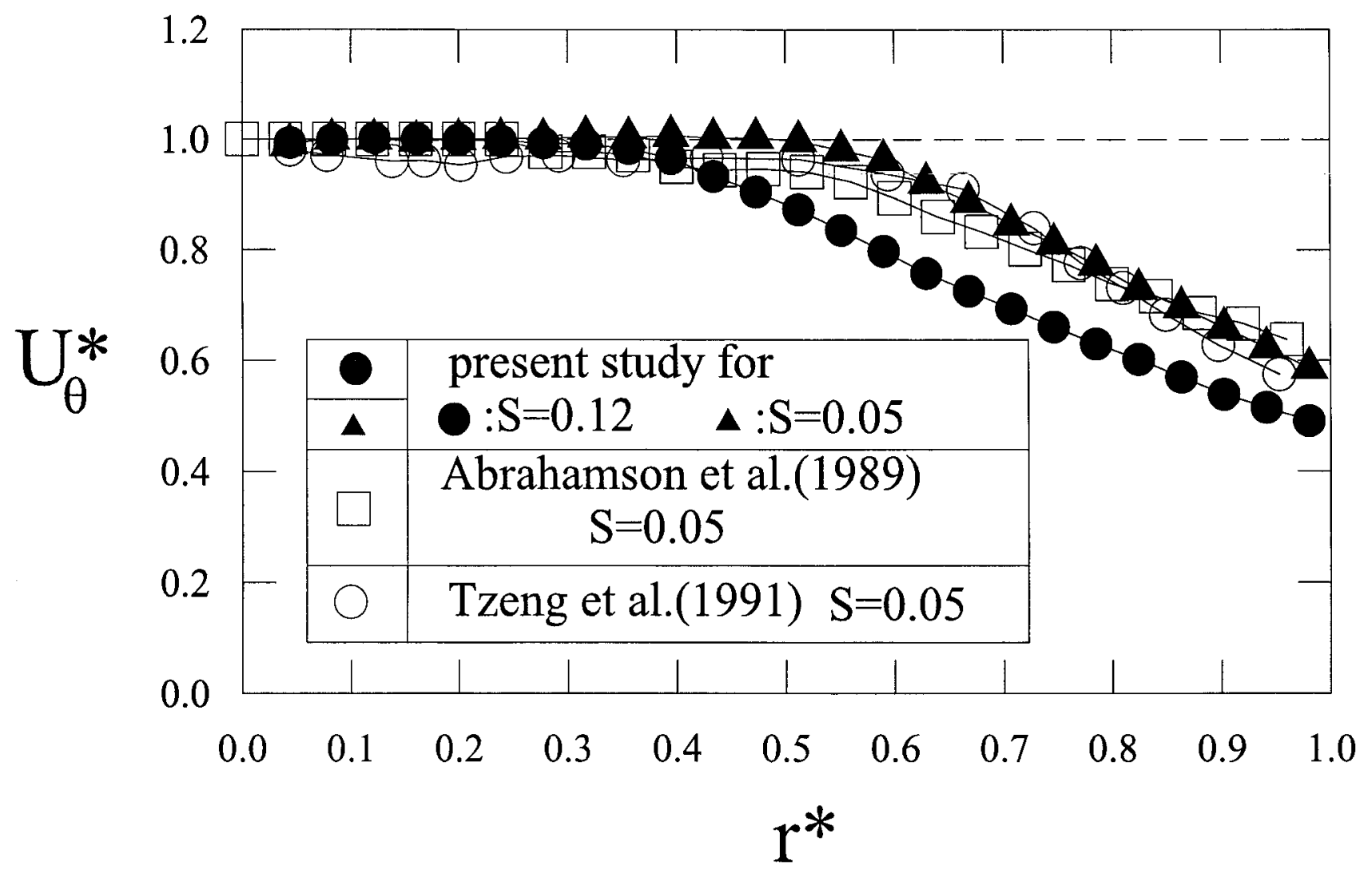

FIGURE 4

Radial profiles of the circumferential mean velocity. 

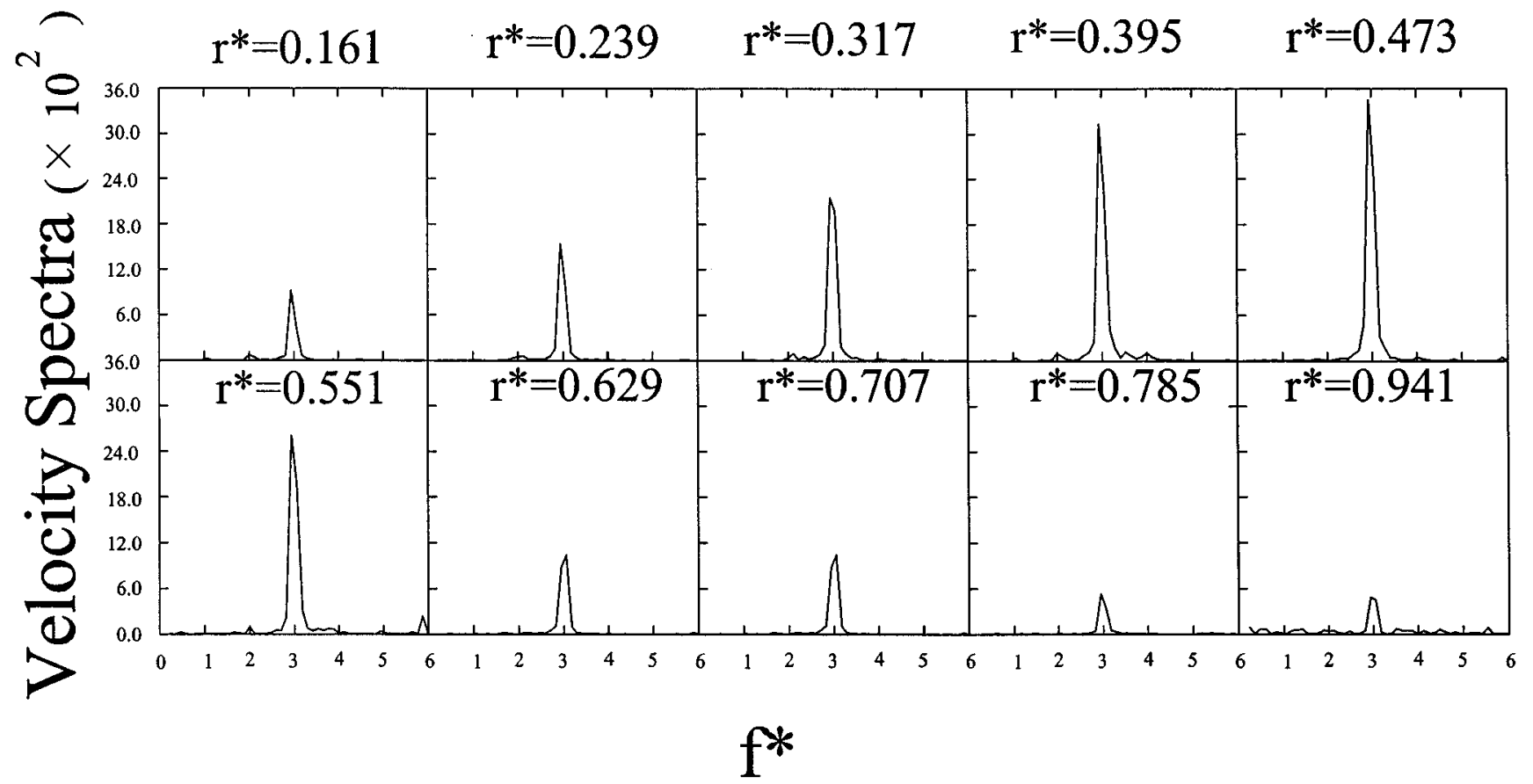

FIGURE 5

Normalized spectra of radial velocity fluctuation at different radial positions.

\section{Power Spectra}

Figure 3 shows the measured time resolved circumferential and radial velocities at the midplane between the two disks at $r^{*}=0.395$. These time sequences display distinct sinusoidal- like oscillations. The Fourier spectra of radial and circumferential velocities at several radial positions were calculated. The results of radial and circumferential velocity power spectra were presented as an example in Figures 5 and 6. The actual

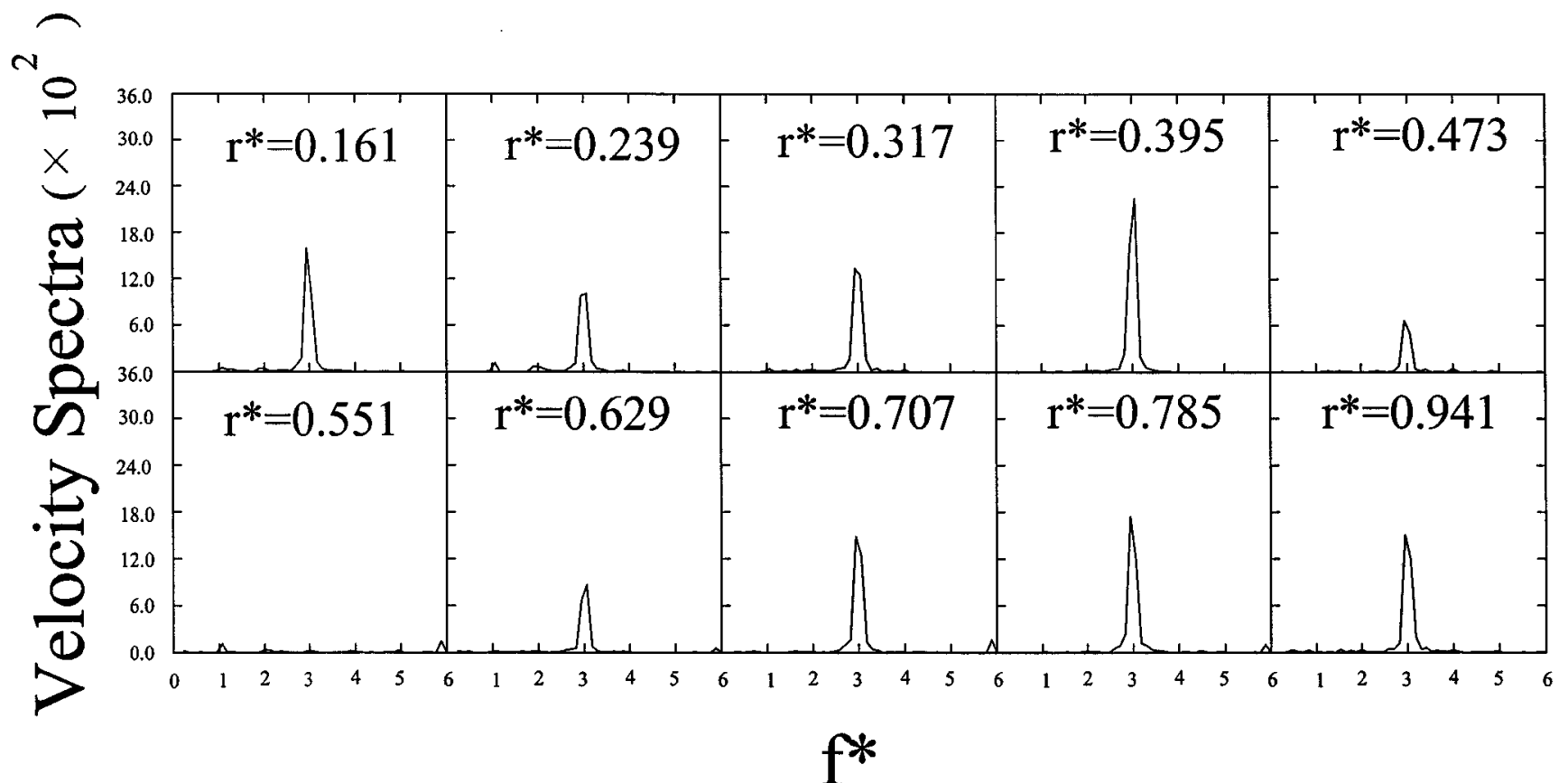

FIGURE 6

Normalized spectra of circumferential velocity fluctuation at different radial positions. 
frequencies of the spectra were normalized by 0.75 times the disk rotational frequency. The spectral results show peaks in energy at integral normalized frequency $f^{*}=3$. Abrahamson, Eaton, and Koga (1989) also reported the similar results.

In addition to the spectral frequency, Figure 5 shows a plot of spectral intensity as a function of radial position. For radial velocity, the spectral intensity increases with $r^{*}$ and reaches a maximum value at about $r^{*}=0.47$, it then begins to decrease to a weak value as $r^{*} \approx 1$. The radial velocity is most energetic at $r^{*} \approx 0.5$, and its spectral intensity displays good symmetry to $r^{*} \approx 0.5$.

It is noted that the distribution of spectral intensity for circumferential velocity (as shown in Figure 6) is quite different to that for radial velocity. There are two local maximums for circumferential power spectra intensity. The first local maximum value takes place near the point $r^{*}=0.4$. It is also the most energetic spot for circumferential velocity since the highest value of the circumferential spectral intensity arises there. It recalls the point at the interface of inner and outer regions as discussed previously. The second local maximum occurs at $r^{*}=0.72$. It is located at the center of vortical structure as can be shown later in Figure 8. From the above discussions, it is clear that the circumferential velocities are more energetic at the interface of inner region and out region and also near the center of vortical structure.

\section{Streamline Patterns at Different Phases}

Figure 7 shows the vector fields (Figure $7(a)$ ) and the streamline pattern (Figure $7(b)$ ) for one of the ten phases. The vector field and streamline pattern as observed with a $0.75 \omega$ reference speed. Figure 8 shows the streamline patterns as observed with a $0.75 \omega$ reference speed. The streamline patterns at different

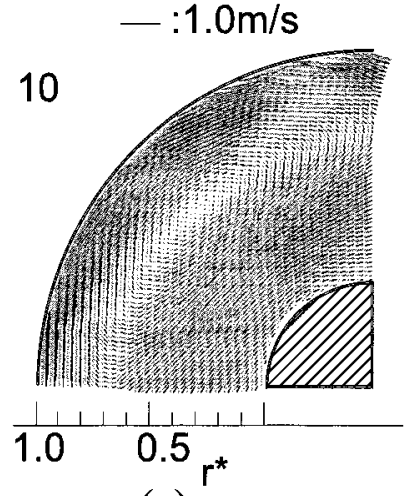

(a)

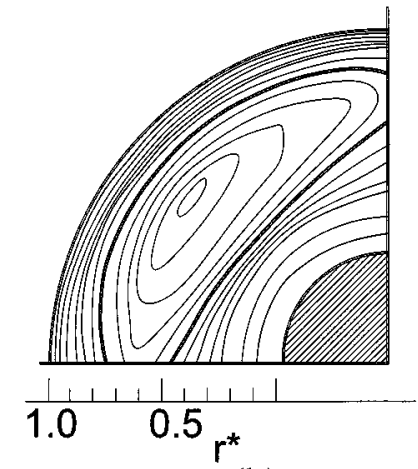

(b)
FIGURE 7

Velocity vector and streamline pattern (a) velocity vector (observed at a reference velocity of $0.75 \omega$ ), (b) streamlines pattern (observed at a reference velocity of $0.75 \omega$ ).

phases during the passage of one vortex are demonstrated as an example. Some other reference speeds were also tested. The test results expose that the vortical structure could not be recognized at a higher reference speed. On the contrary, the vortical structure locates in solid-body rotation region at a lower reference speed. This above phenomenon is obviously unreasonable. The appropriate rotational speed was found to be $(0.75 \pm 0.05) \omega$ in the present case. Similar reference velocity was also found by other researchers, such as Abrahamson et al. (1989). In their work, the camera was mounted to a motor for taking pictures, which followed the rotation of the disks. The vortical structure could not be observed under fixed reference frame. Three distinct regions can be distinguished from the streamline patterns in Figure 8. The inner solid-body rotation region ranges from
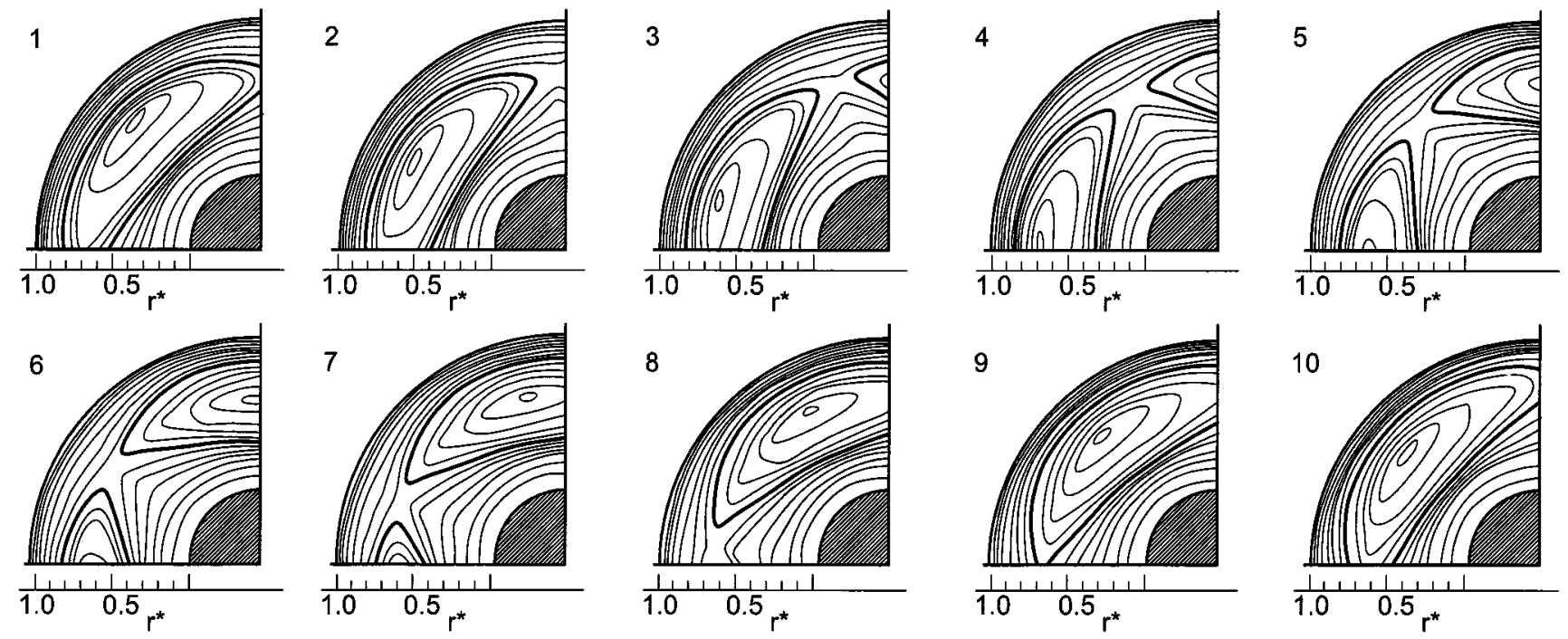

FIGURE 8

The streamline patterns at different phase (reference velocity at $0.75 \omega$, contour interval 0.2 ). 
$r^{*} \cong 0$ to 0.35 ; the outer vortical structure region from $r^{*} \cong 0.35$ to 0.9 ; the shrouded boundary-layer region from $r^{*}=0.9$ to 1 . It is noted that there seems to be no radial flow across the boundary between the rotating disks and the outside of the disks, as shown in Figure 8. However, due to the centrifugal force acting on the rotating fluid, there must exist radial inflow and outflow. The inflow and outflow regions rotate in the same direction as the disks forming a periodic flow field (Mochizuki, 1993). Extended experiments have been carried out in the crossstream $(r-z)$ plane $(\mathrm{Wu}, 2000)$. The experimental results show that there is indeed radial flow. However, the circumferential components are much larger than the radial ones, therefore, the radial flow is not clearly observed from the phase-average measured data.

Although only a quarter of the frame was reconstructed, the number of the vortical structure can be easily determined to be three. This integer is the same as the normalized frequency found in the power spectra analysis, as shown in Figures 5 and 6 . These findings demonstrate that the velocity oscillations result from the periodic passage of flow structure. The center of vortex is located at $r^{*}=0.72$. In addition, the radius of inscribe circle of the vortical structure is found to be the same as that calculated by the formula $R_{i}=R_{2}\left(\frac{N-\pi A}{N+\pi A}\right)$ proposed by Tzeng, Munce, and Crawforth (1991), where $\pi \mathrm{A}=N-f_{s} / \omega$. The oscillatory frequency $f_{s}$ measured is $9.9 \mathrm{~Hz}$, and the number of vortical structure $N$ is 3 in the present study. The inscribe circle radius calculated by the formula is thereby $R i=108.7 \mathrm{~mm}$. In Figure 7, the inscribe circle of each phase was drawn to find its radius and the average value of the ten radii was taken. The inscribe circle of the polygonal structure with the average radius intersect the dimensionless radial position at $r^{*}=\left(r-R_{1}\right) /\left(R_{2}-R_{1}\right)=0.4$, corresponding to $r=R_{i}=110.7 \mathrm{~mm}$. There is little difference between the calculated and measured values.

\section{CONCLUSIONS}

Major conclusions are summarized as below:

1. By using two laser Doppler velocimeters and phaseaveraged method, temporal vortical structure in the flow between co-rotating disks could be quantitatively reconstructed. The detailed flow information, including the mean, periodic, and random terms provides a basis for numerical verification.

2. The power spectral analysis showed all peaks in the energy are at same integral normalized frequency. The value of the normalized frequency is the same as the number of vortical structure observed in the reconstructed streamline patterns. This indicates that the flow oscillations result from the periodic passage of flow structure.

3. From the reconstructed streamline patterns, three distinct regions could be quantitatively identified: namely, an inner region near the hub, an outer region, and a shroud boundary-layer region.

\section{NOMENCLATURE}

a distance between disk tip and shroud

$b \quad$ thickness of disks

$f^{*} \quad$ normalized frequency

$f_{\mathrm{s}} \quad$ dominate frequency of power spectra

$H \quad$ axial distance between disks

$N \quad$ numbers of polygonal structures

$r \quad$ radial coordinates for the system

$r^{*} \quad$ dimensionless radial coordinates $=\left(r-R_{1}\right) /\left(R_{2}-R_{1}\right)$

$R_{1} \quad$ radius of hub

$R_{2} \quad$ radius of disks

Re Reynolds number $=R_{2}^{2} \omega / v$

$R_{i} \quad$ radius of inscribing circle of polygon structure

$S \quad$ dimensionless axial distance of disks $=H / R_{2}$

$U, u$ velocity

$U_{r} \quad$ radial velocity component

$U_{\text {ref }}$ circumferential velocity of the reference point

$U_{\theta} \quad$ circumferential velocity component

$U_{\theta}{ }^{*} \quad$ normalized circumferential velocity $=U_{\theta} / r \omega$

$\bar{u} \quad$ global mean of $u$

$\tilde{u} \quad$ periodic fluctuation away from $\bar{u}$

$u^{\prime} \quad$ random fluctuation away from $\tilde{u}$

$Z \quad$ axial coordinates for the system

$\theta \quad$ circumferential coordinates for the system

$v \quad$ kinematic viscosity

$\omega \quad$ rotating speed of disks

\section{REFERENCES}

Abrahamson, S. D., Koga, D. J., and Eaton, J. K. 1988. Flow visualization and spectral measurements in a simulated rigid disk drive. IEEE Trans. Comp., Hybrids and Manuf. Tech. 11(4):576-584.

Abrahamson, S. D., Eaton, J. K., and Koga, D. J. 1989. The flow between shrouded co-rotating disks. Phys. Fluids A. 1(2):241-251.

Abrahamson, S. D., Eaton, J. K., and Chang, C. 1991. Flow structure in head-disk assemblies and implications for design. Adv. Info. Storage Syst. 1:111-132.

Durst, F., Melling, A., and Whitelaw, J. H. 1981. Principles and Practice of Laser-Doppler Anemometry. London: Academic.

Humphrey, J. A. C., and Gor, D. 1993. Experimental observation of an unsteady detached shear layer in enclosed co-rotating disk flow. Phys. Fluids 5:2438.

Humphrey, J. A. C., Schuler, C. A., and Weber, D. R. 1995. Unsteady laminar flow between a pair of disks co-rotating in a fixed cylindrical enclosure. Phys. Fluids 7:1225-1240.

Hussain, A. K. M. F., and Reynolds, W. C. 1970. The mechanics of an organized wave in turbulent shear flow. J. Fluid Mech. 41:241-258.

Kaneko, R., Oguchi, S., and Hoshiya, K. 1977. Hydrodynamic characteristics in disk packs for magnetic storage. Rev. Elec. Comm. Lab. 25(11):1325-1336.

Lennemann, E. 1974. Aerodynamic aspects of disk files. IBM J. Res. Develop. 18(6):480-488.

McLaughlin, D. K., and Tiederman, W. G. 1973. Biasing correction for individual realization of laser anemometer measurements in turbulent flows. Phys. Fluids 16(12):2082-2088.

Mochizuki, S. 1993. Unsteady flow phenomena and heat transfer in rotating-disk systems. Proc. 6th International Symposium on 
Transport Phenomena in Thermal Engineering, Seoul, Korea, May, 279-289.

Reynolds, W. C., and Hussain, A. K. M. F. 1972. The mechanics of an organized wave in turbulent shear flow. Part 3. Theoretical models and comparisons with experiments. J. Fluid Mech. 54:263288.

Schuler, C. A., Usry, W., Weber, B., Humphrey, J. A. C., and Greif, R. 1990. On the flow in the unobstructed space between shrouded co-rotating disks. Phys. Fluids A 2(10):1760-1770.
Tzeng, H. M., Munce, A. C., and Crawforth, L. 1991. Quantitative airflow visualization between shrouded co-rotating disks. ASME Exp. Num. Flow Visualization 128:141-147.

Tzeng, H. M., and Humphrey, J. A. C. 1991. Co-rotating disk flow in an axisymmetric enclosure with and without a bluff-body. Int. J. Heat and Fluid Flow 12(3):194-201.

$\mathrm{Wu}$, S. C. 2000. The flow between corotating disks with/without an obstruction in a cylindrical enclosure. Ph.D. diss., National Taiwan University. 

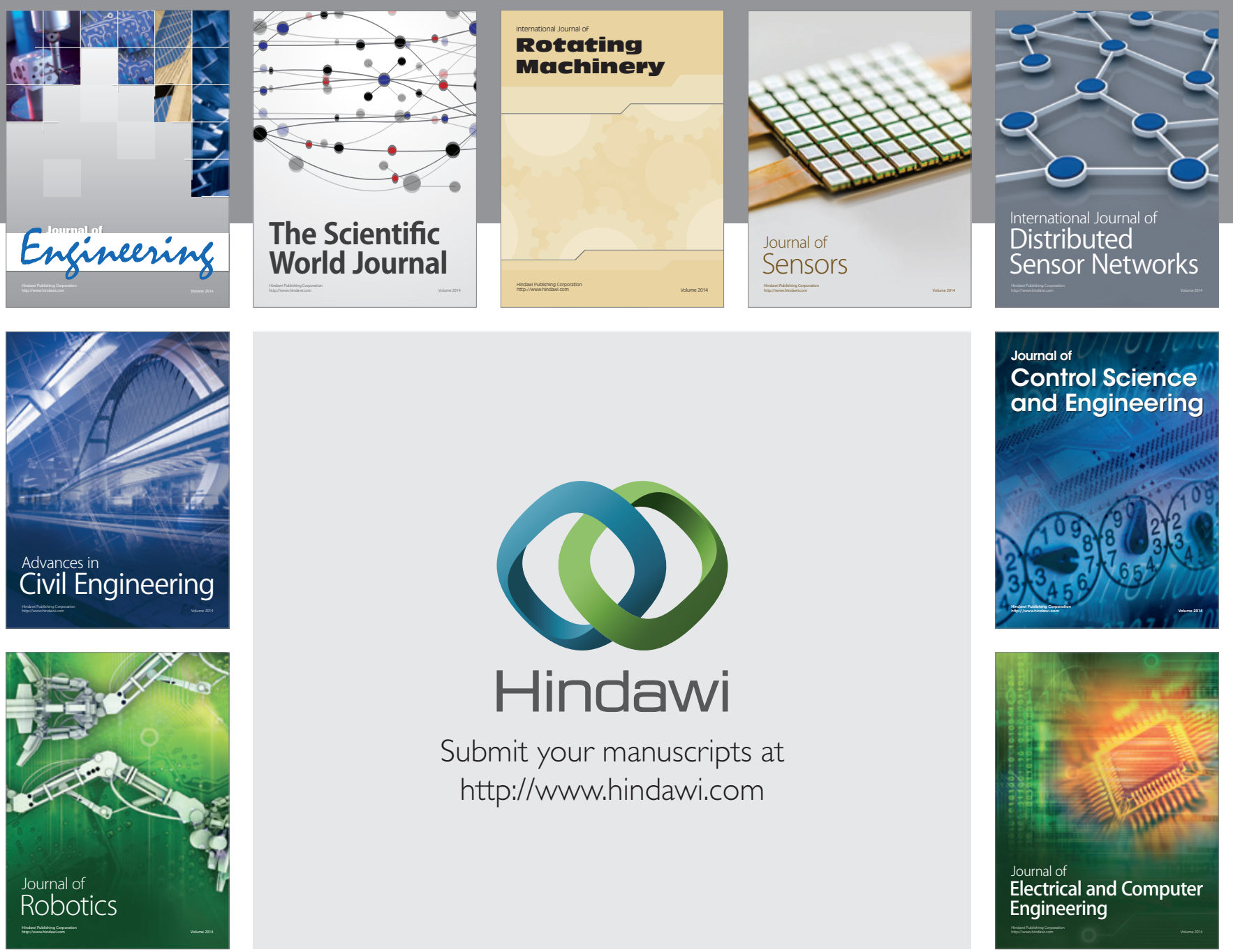

Submit your manuscripts at

http://www.hindawi.com
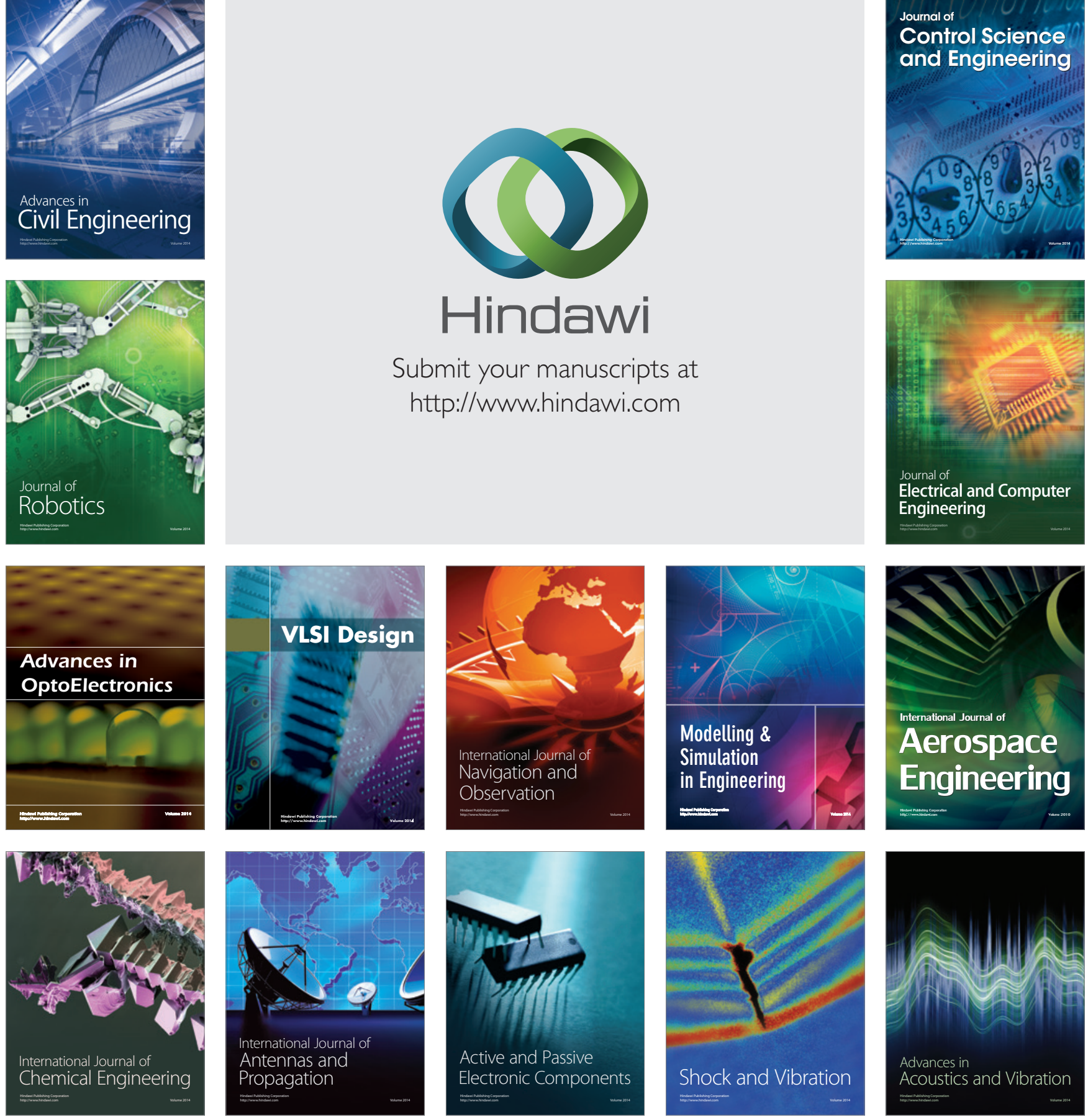